Supporting Information

\title{
Atomic PdAu Interlayer Sandwiched into Pd/Pt Core/Shell Nanowires Achieves Superstable Oxygen Reduction Catalysis
}

Lu Tao, ${ }^{1,2}$ Bolong Huang, ${ }^{*, 3}$ Fengdan Jin, ${ }^{1}$ Yong Yang, ${ }^{2}$ Mingchuan Luo, ${ }^{2}$ Mingzi Sun, ${ }^{3}$ Qian Liu, ${ }^{1}$ Faming Gao ${ }^{*, 1}$ and Shaojun Guo*,2

${ }^{1}$ Key Laboratory of Applied Chemistry, College of Environmental and Chemical

Engineering, Yanshan University, Qinhuangdao 066004, P. R. China.

${ }^{2}$ Department of Materials Science \& Engineering, \& BIC-ESAT, College of Engineering, Peking University, Beijing, 100871, China.

${ }^{3}$ Department of Applied Biology and Chemical Technology, the Hong Kong Polytechnic University, Hung Hom, Kow-loon, Hong Kong SAR, China.

\section{Corresponding Author}

*E-mail: bhuang@polyu.edu.hk; fmgao@ysu.edu.cn; guosj@pku.edu.cn 
This PDF file includes:

1. Figures $\mathrm{S} 1-11$

2. Table 1

3. Reference 


\section{Figures}
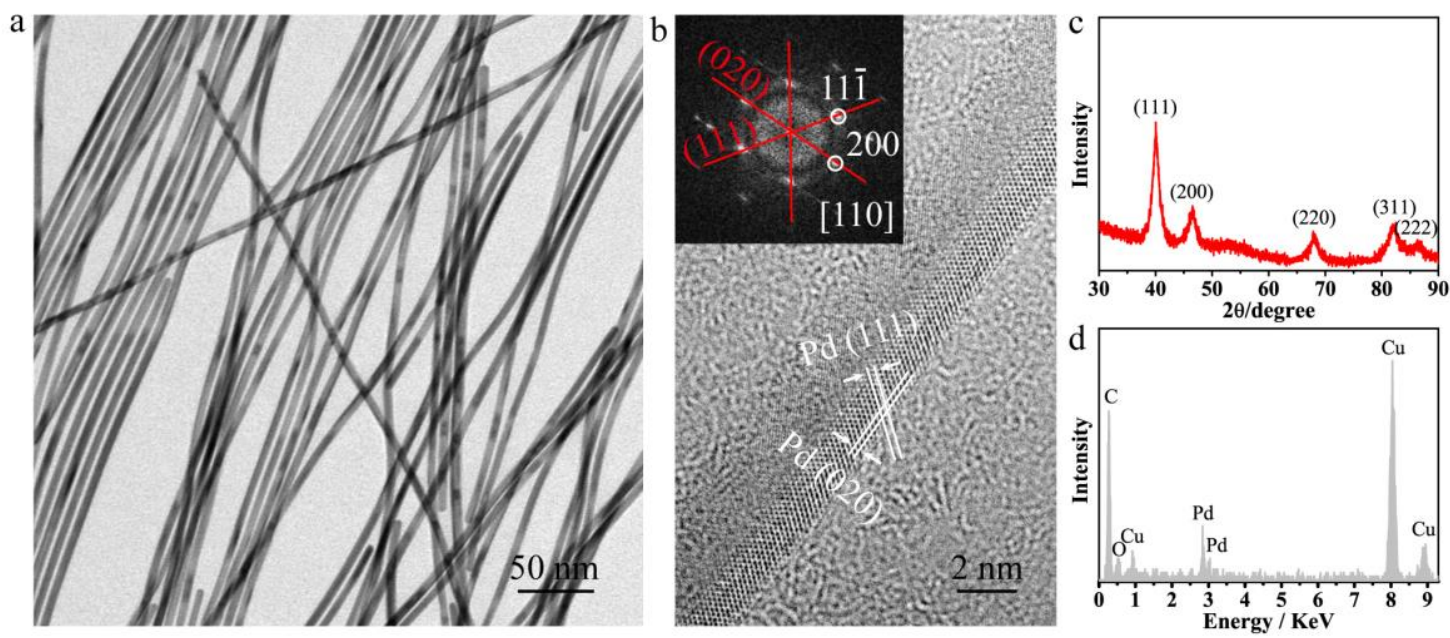

Figure S1. (a) TEM image of Pd NWs. (b) HRTEM image of one Pd NW and corresponding Fast Fourier Transformed (FFT) image in the inset. (c) XRD and (d) EDS pattern of Pd NWs. 

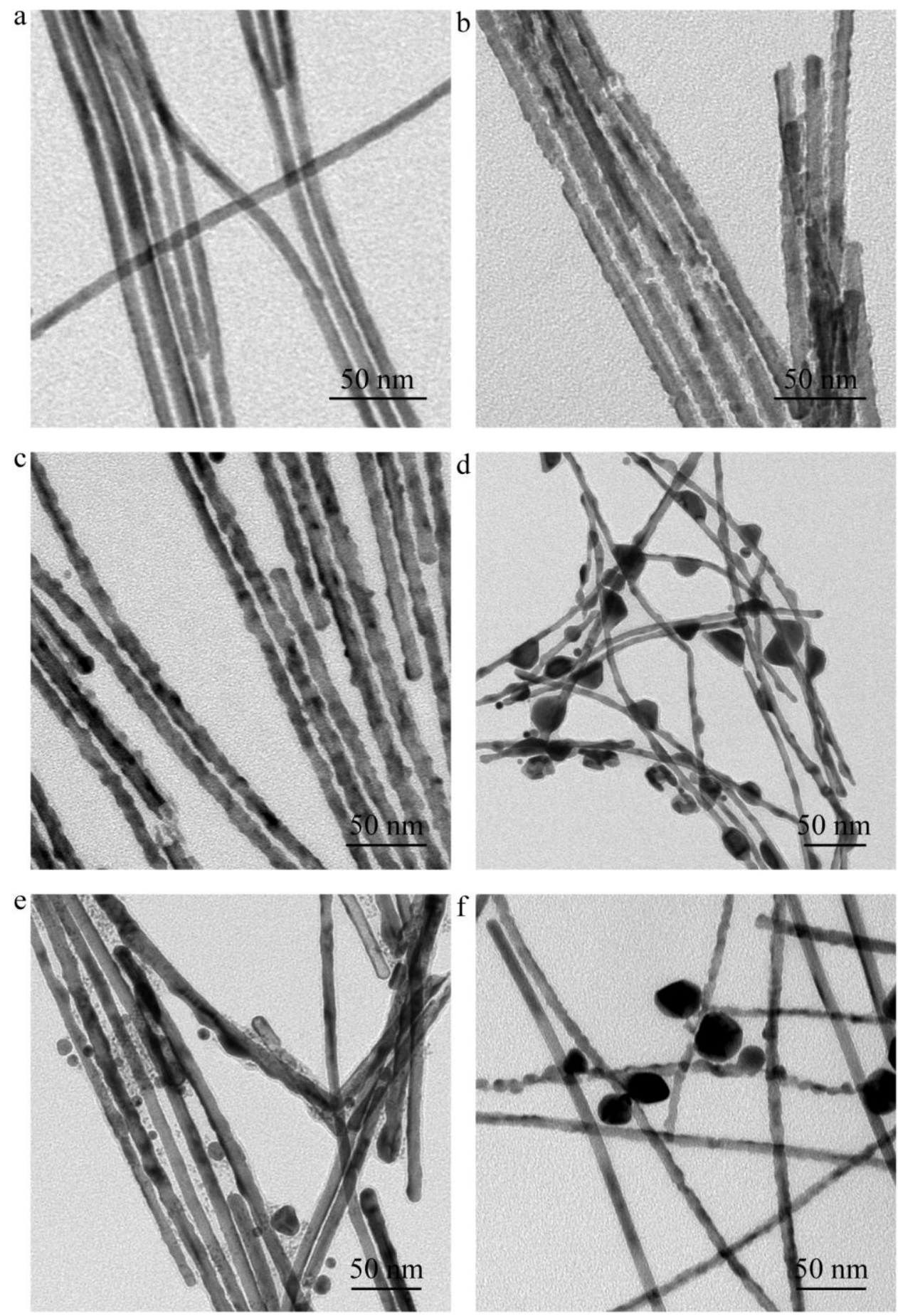

Figure S2. TEM images of PdAu nanostructures obtained by reacting Pd NWs with $\mathrm{HAuCl}_{4}$ in (a) ethylene glycol, (b) benzyl alcohol, (c) water, (d) ethanol, (e) OAm and (f) OA, respectively. 

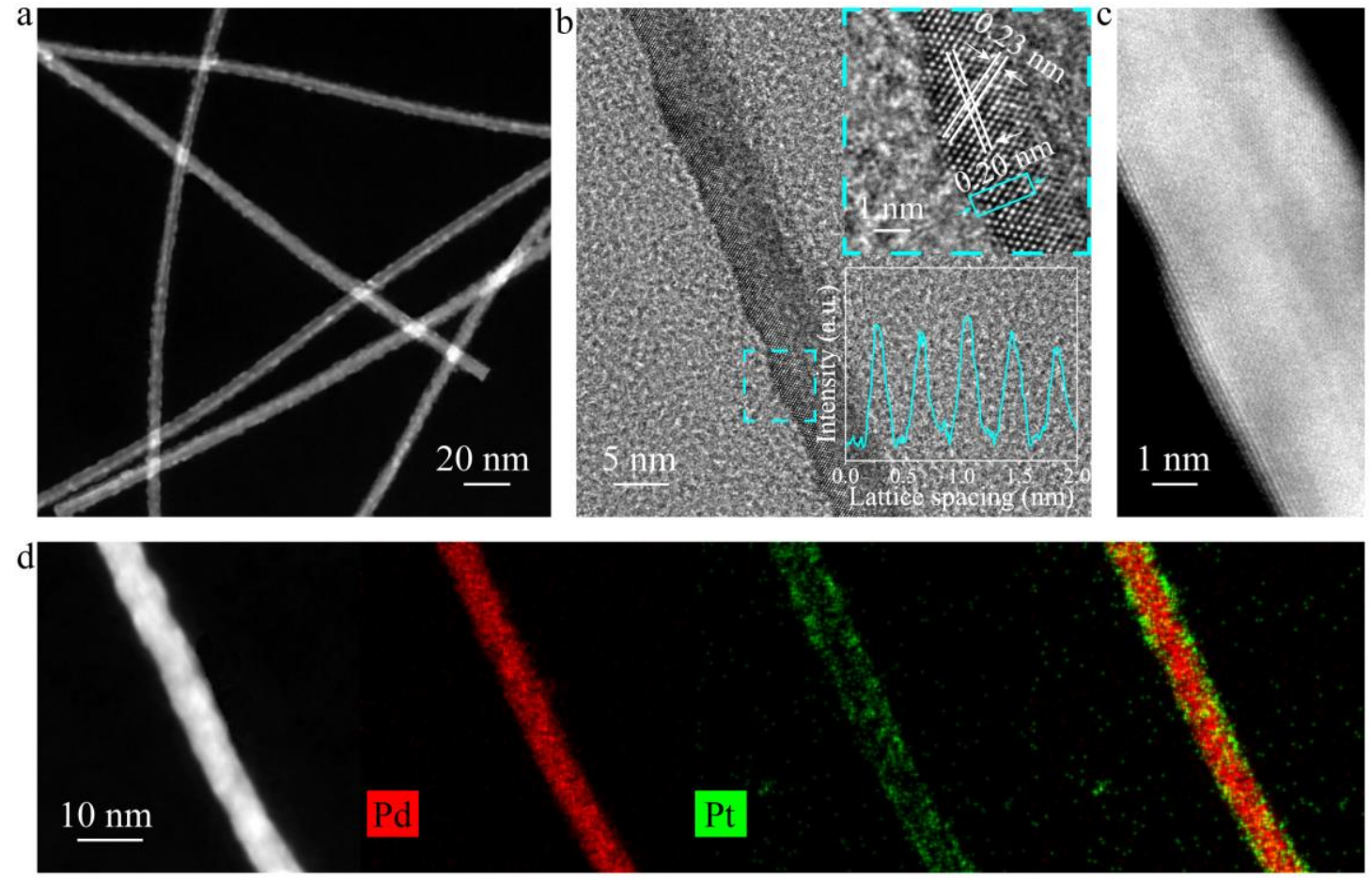

Figure S3. (a) STEM image of Pd/Pt NWs. (b) HRTEM image of a segment Pd/Pt NW (inset: enlarged HRTEM image from the dashed square area and the integrated pixel intensities of $\mathrm{Pd} / \mathrm{Pt}$ taken from the cyan solid rectangle region along the arrow direction. (c) HAADF-STEM image of surface atomic arrangement on the Pd/ Pt NW (d) STEM elemental mapping of one $\mathrm{Pd} / \mathrm{Pt} \mathrm{NW}$. The obtained $\mathrm{Pd} / \mathrm{Pt} \mathrm{NWs}$ were about $6 \mathrm{~nm}$ in diameter and several microns in length. 

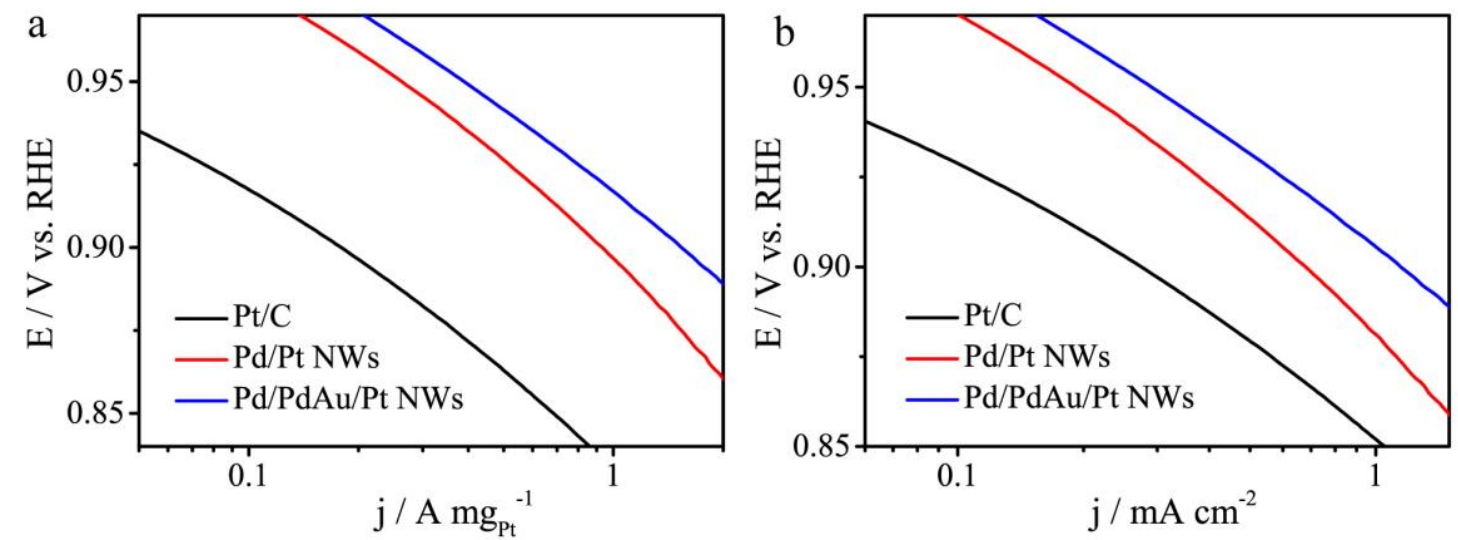

Figure S4. (a) MAs and (b) SAs Tafel plots of the Pd/PdAu/Pt NWs, Pd/Pt NWs and commercial $\mathrm{Pt} / \mathrm{C}$ catalysts. 

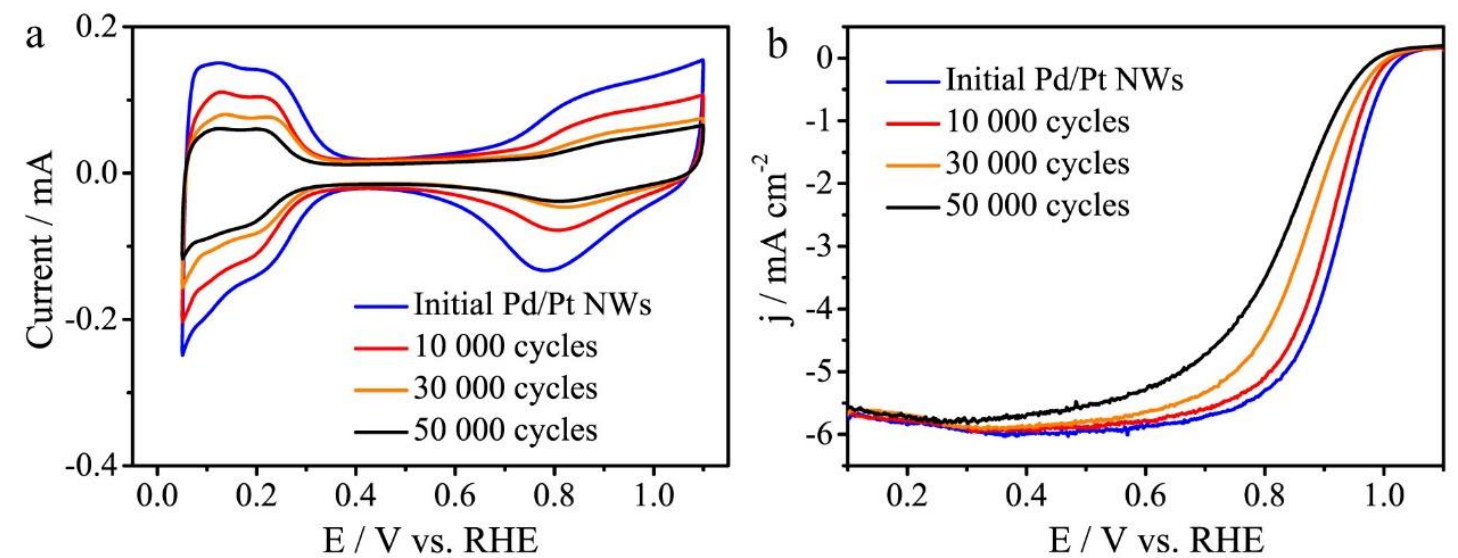

Figure S5. (a) CVs and (b) ORR polarization curves of Pd/Pt NWs before and after 10 000, 30000 and 50000 potential cycles between $0.6 \mathrm{~V}$ and $1.1 \mathrm{~V} v s$. RHE. 

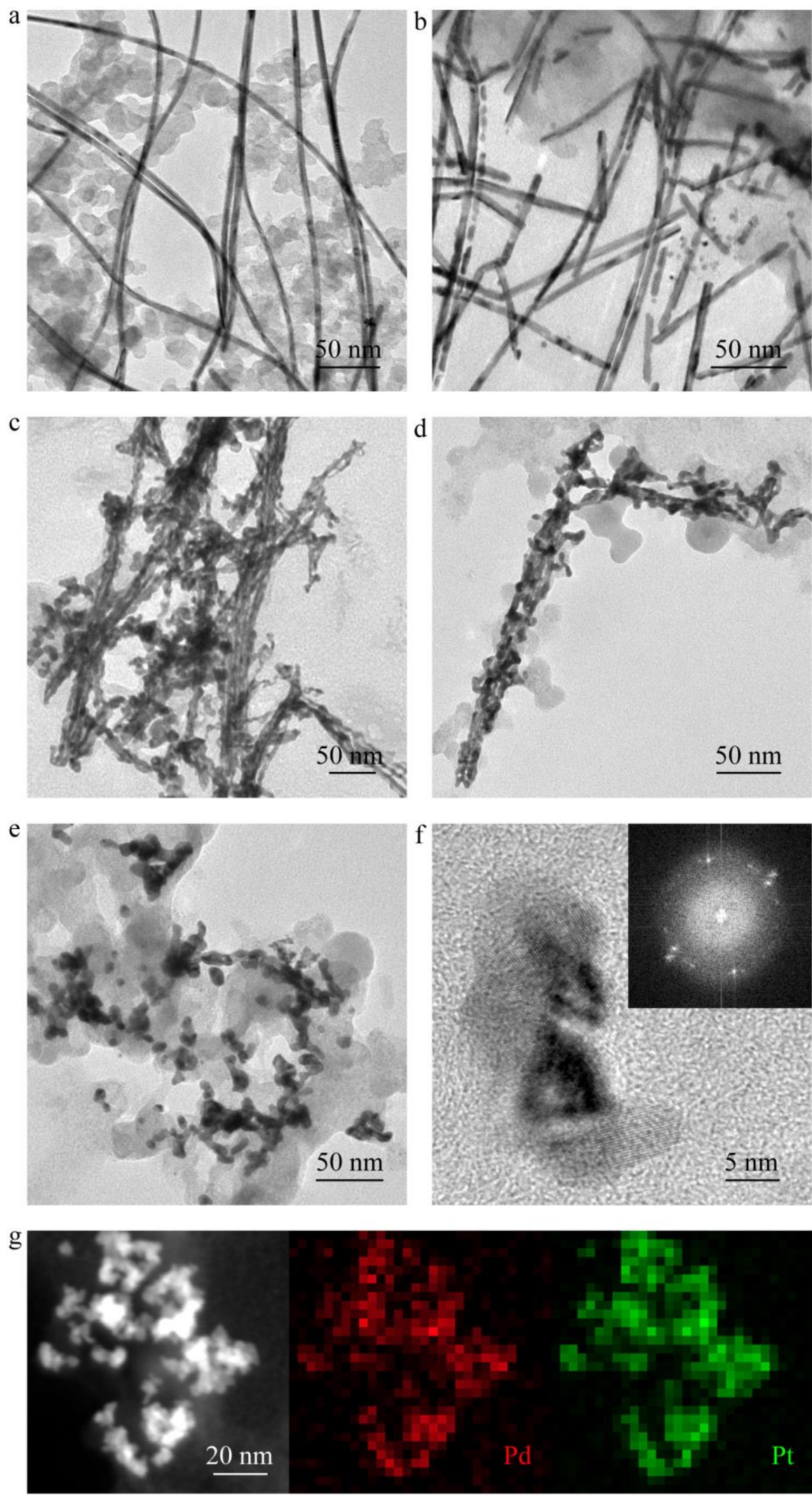

Figure S6. TEM image of the Pd/Pt NWs/C before (a) and after 10000 (b), 30000 (c), 50000 (d) and 80000 (e) potential cycles between $0.6 \mathrm{~V}$ and $1.1 \mathrm{~V} v s$. RHE. HRTEM (f) and STEM-EDS elemental mapping (g) of the Pd/Pt NWs after 80000 potential cycles between $0.6 \mathrm{~V}$ and $1.1 \mathrm{~V} v s$. RHE. 

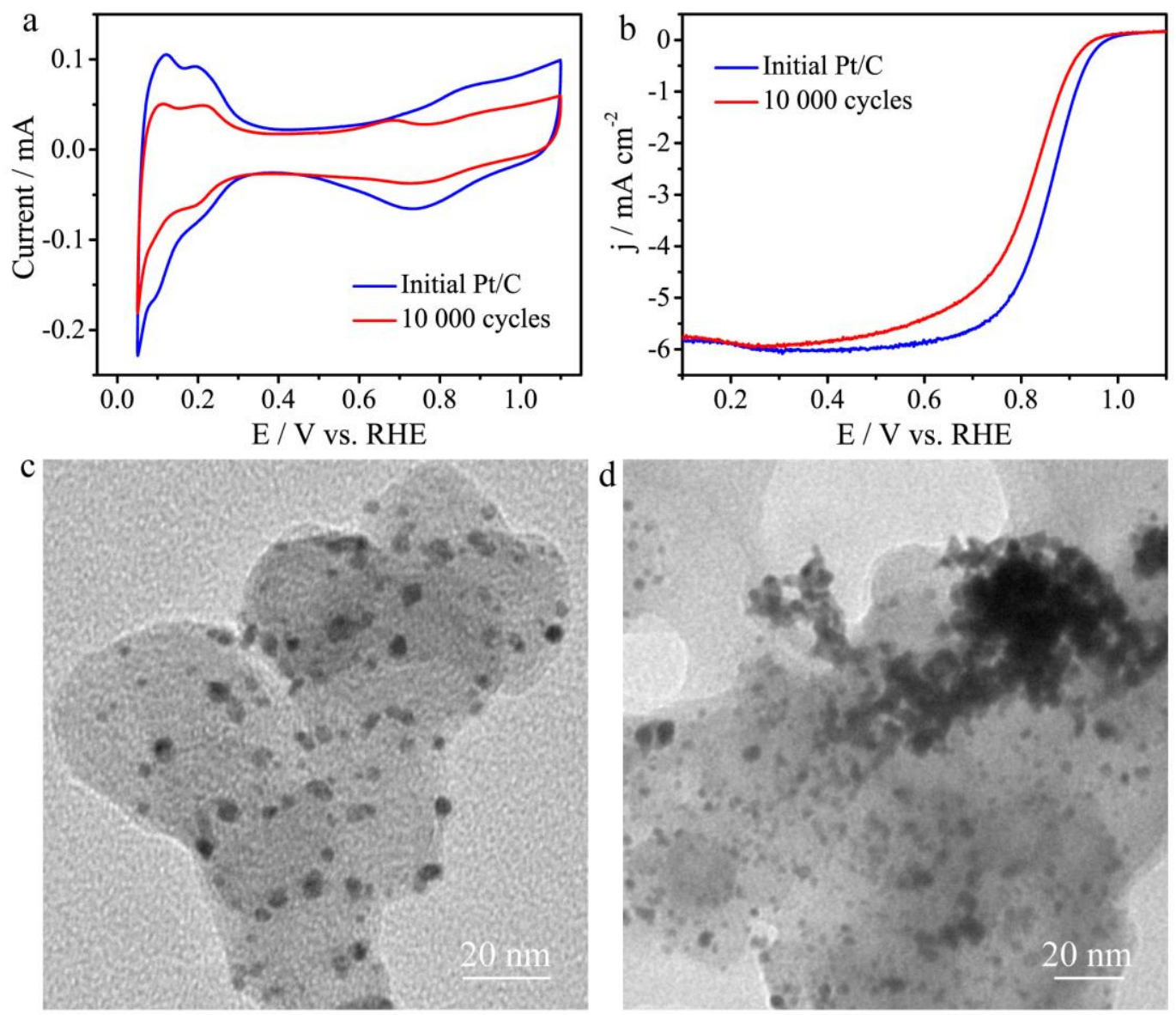

Figure S7. (a) CVs and (b) ORR polarization curves of commercial $\mathrm{Pt} / \mathrm{C}$ before and after 10000 potential cycles between $0.6 \mathrm{~V}$ and $1.1 \mathrm{~V} v$ s. RHE. TEM image of commercial Pt/C before (c) and after 10000 (d) potential cycles between $0.6 \mathrm{~V}$ and 1.1 V vs. RHE. 

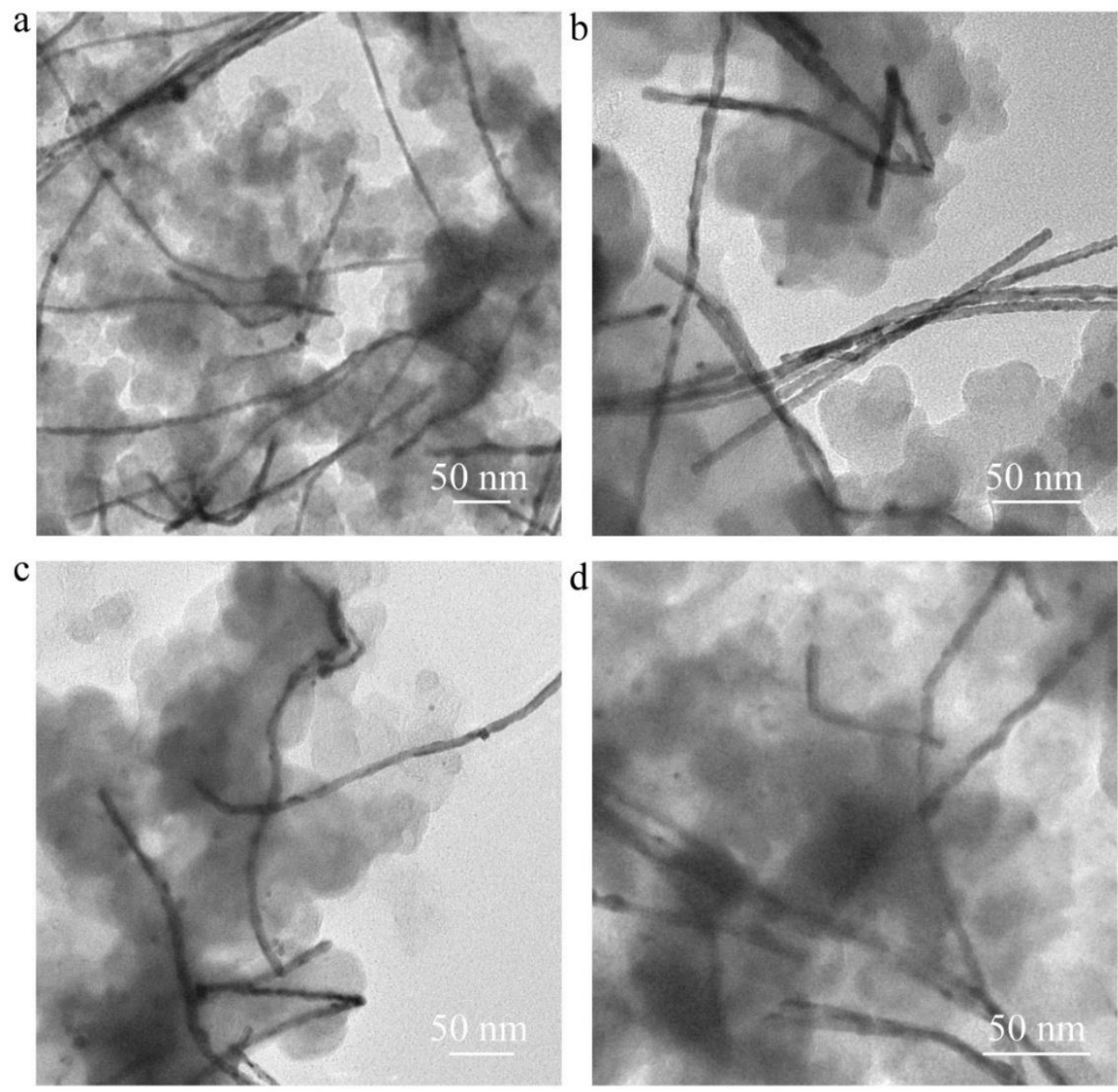

Figure S8. TEM image of the Pd/PdAu/Pt NWs/C after 10000 (b), 30000 (c), 50000 (d) and 80000 (e) potential cycles between $0.6 \mathrm{~V}$ and $1.1 \mathrm{~V}$ vs. RHE. 

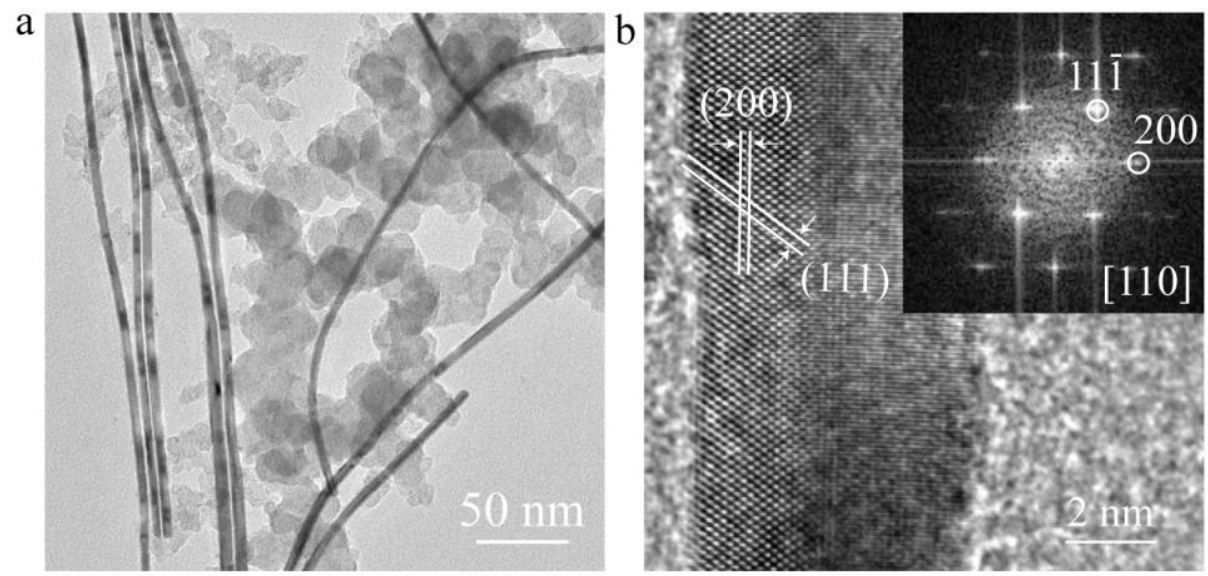

Figure S9. (a) TEM image of the initial Pd/PdAu/Pt NWs/C. (b) HRTEM image of one $\mathrm{Pd} / \mathrm{PdAu} / \mathrm{Pt} \mathrm{NW}$ and corresponding FFT image in the inset. The calculated average diameter of $\mathrm{Pd} / \mathrm{PdAu} / \mathrm{Pt} \mathrm{NWs}$ was about $7 \mathrm{~nm}$. 

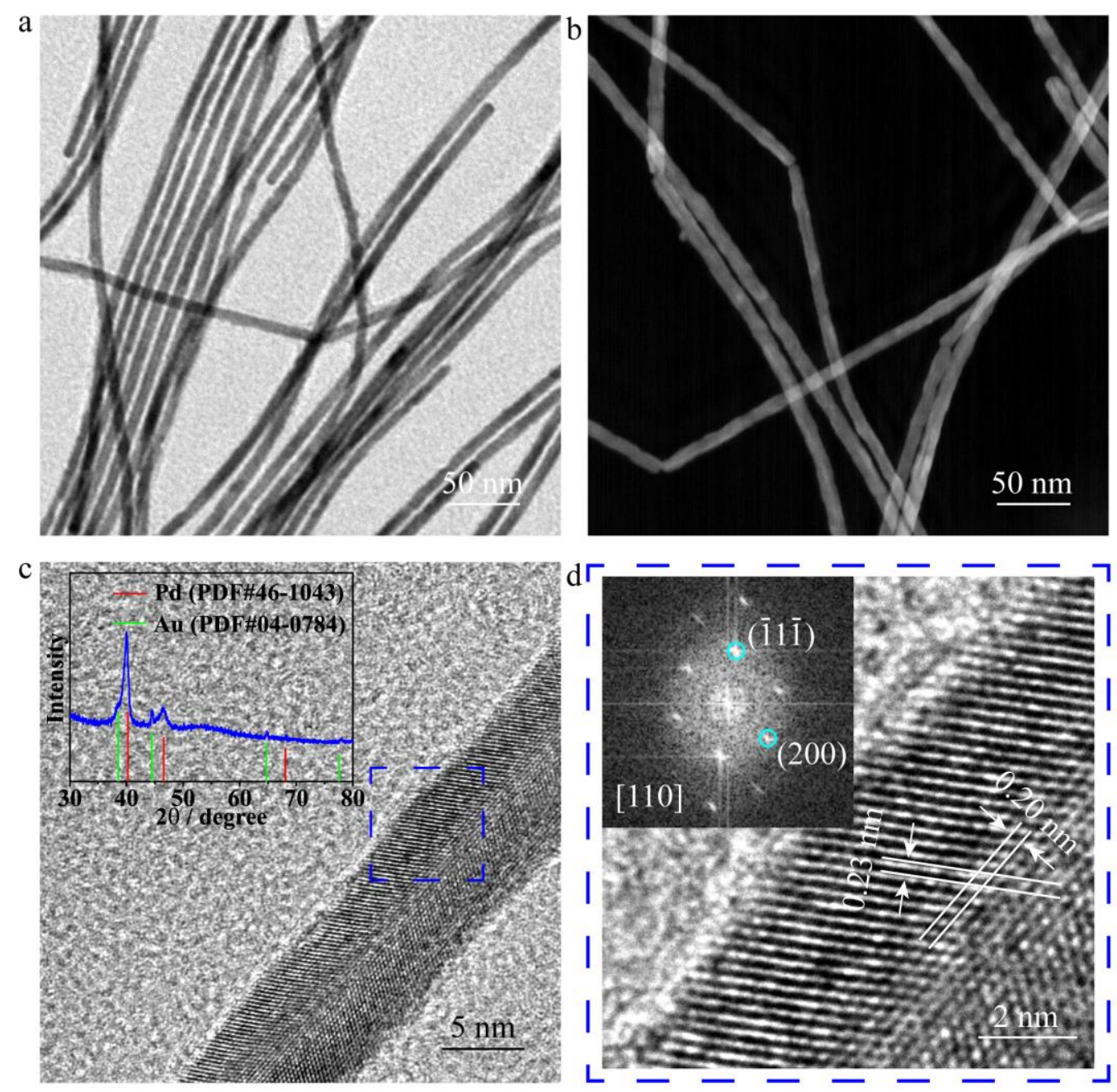

Figure S10. (a) TEM and (b) STEM images of Pd/PdAu NWs. (c) HRTEM image of a segment $\mathrm{Pd} / \mathrm{PdAu} \mathrm{NW}$ (inset: XRD pattern of Pd/PdAu NWs). (d) Enlarged HRTEM image of the $\mathrm{Pd} / \mathrm{PdAu} \mathrm{NW}$ as boxed in (c) (inset: corresponding FFT). The as-prepared $\mathrm{Pd} / \mathrm{PdAu} \mathrm{NWs}$ were about $6 \mathrm{~nm}$ in diameter and several microns in length. 

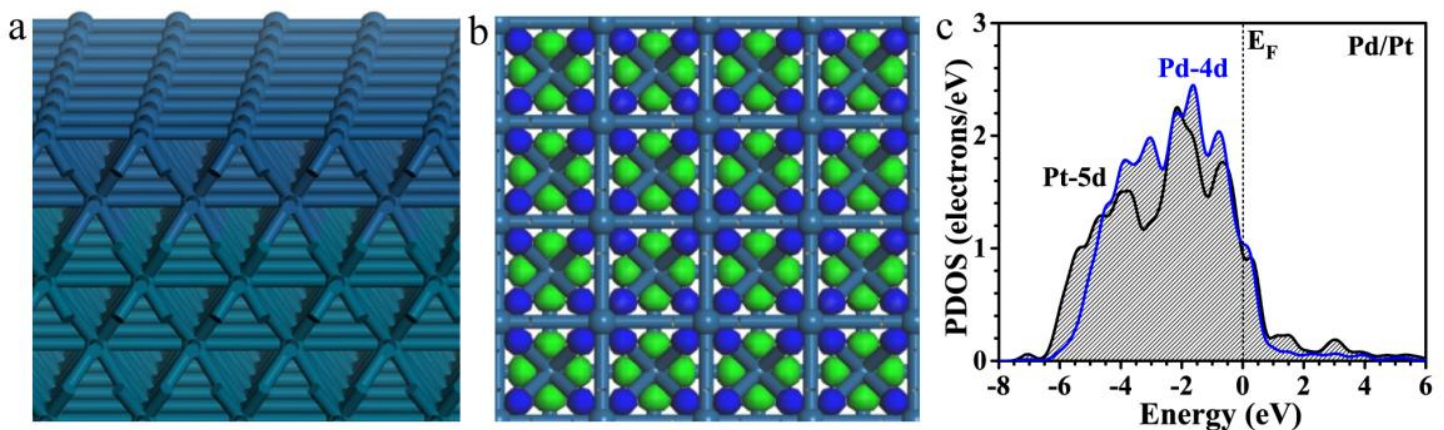

Figure S11. The electronic properties of $\mathrm{Pd} / \mathrm{Pt}$. (a) The core/shell structure of $\mathrm{Pd} / \mathrm{Pt}$ and (b) the bonding and anti-bonding orbital distribution near the Fermi level $\left(\mathrm{E}_{\mathrm{F}}\right)$. (c) The PDOS of Pd/Pt. 
2. Table 1. Heterostructures for ORR in $0.1 \mathrm{M} \mathrm{HClO}_{4}$ acidic electrolyte.

\begin{tabular}{|c|c|c|c|c|c|}
\hline Catalyst & Morphology & $\begin{array}{c}\text { MA } \\
\left(\mathrm{A} / \mathrm{mg}_{\mathrm{Pt}}\right)\end{array}$ & $\begin{array}{c}\mathrm{SA} \\
\left(\mathrm{mA} / \mathrm{cm}^{2}\right)\end{array}$ & Stability & Reference \\
\hline $\mathrm{Pd} / \mathrm{PdAu} / \mathrm{Pt}$ & $\begin{array}{c}\mathrm{Pd} \text { core } / \mathrm{PdAu} \\
\text { interlayer/Pt } \\
\text { shell } \\
\text { nanowires }\end{array}$ & 1.54 & 1.15 & $\begin{array}{l}\text { After } 80000 \text { cycles, } \\
\text { the MA decreased to } \\
1.42 \mathrm{~A} / \mathrm{mg}_{\mathrm{Pt}}\end{array}$ & This work \\
\hline $\mathrm{Au} / \mathrm{FePt}_{3}$ & $\begin{array}{c}\text { Bimetallic } \\
\text { PtFe on } \mathrm{Au} \\
\text { nanoparticles }\end{array}$ & 0.54 & 1.50 & $\begin{array}{l}\text { After } 60000 \text { cycles, } \\
\text { the MA decreased to } \\
0.47 \mathrm{~A} / \mathrm{mg}_{\mathrm{Pt}}\end{array}$ & [1] \\
\hline $\mathrm{Au} / \mathrm{Pt}$ & $\begin{array}{l}\text { Monolayer-Pt } \\
\text { on } \mathrm{Au} \\
\text { nanoparticles }\end{array}$ & 1.20 & 0.51 & $\begin{array}{l}\text { After } 5000 \text { cycles, } \\
\text { the MA decreased to } \\
0.90 \mathrm{~A} / \mathrm{mg}_{\mathrm{Pt}}\end{array}$ & [2] \\
\hline $\mathrm{Pd}_{9} \mathrm{Au}_{1} / \mathrm{Pt}$ & $\begin{array}{c}\text { Pt on PdAu } \\
\text { nanoparticles }\end{array}$ & 0.31 & 0.38 & $\begin{array}{c}\text { After } 100000 \\
\text { cycles, the MA } \\
\text { decreased to } 0.29 \\
\mathrm{~A} / \mathrm{mg}_{\mathrm{Pt}}\end{array}$ & [3] \\
\hline $\mathrm{AuCu} / \mathrm{Pt}$ & $\begin{array}{c}\mathrm{Pt} \text { on } \mathrm{AuCu} \\
\text { nanoparticles }\end{array}$ & 0.56 & 0.37 & $\begin{array}{l}\text { After } 10000 \text { cycles, } \\
\text { the MA decreased to } \\
\quad 0.40 \mathrm{~A} / \mathrm{mg}_{\mathrm{Pt}}\end{array}$ & [4] \\
\hline $\begin{array}{c}\mathrm{Au} / \mathrm{Pt} \\
\text { decahedra }\end{array}$ & $\begin{array}{c}\mathrm{Au} / \mathrm{Pt} \\
\text { core/shell } \\
\text { decahedra }\end{array}$ & 0.94 & 1.09 & $\begin{array}{l}\text { After } 30000 \text { cycles, } \\
\text { the MA decreased to } \\
\quad 0.75 \mathrm{~A} / \mathrm{mg}_{\mathrm{Pt}}\end{array}$ & [5] \\
\hline $\mathrm{Au} / \mathrm{NiPt}$ & $\begin{array}{c}\text { PtNi alloys } \\
\text { on } \mathrm{Au} \\
\text { nanoparticles }\end{array}$ & 0.56 & 0.80 & $\begin{array}{l}\text { After } 20000 \text { cycles, } \\
\text { the MA decreased to } \\
\quad 0.40 \mathrm{~A} / \mathrm{mg}_{\mathrm{Pt}}\end{array}$ & [6] \\
\hline $\mathrm{AuNi} / \mathrm{Pt}$ & $\begin{array}{l}\text { Monolayer-Pt } \\
\text { on AuNi } \\
\text { nanoparticls }\end{array}$ & 1.52 & 1.18 & $\begin{array}{l}\text { After } 5000 \text { cycles, } \\
\text { the MA decreased to } \\
1.47 \mathrm{~A} / \mathrm{mg}_{\mathrm{Pt}}\end{array}$ & [7] \\
\hline $\mathrm{Pt}_{\mathrm{n}} \mathrm{Au}_{100-\mathrm{n}}$ & $\begin{array}{c}\mathrm{Pt}_{76} \mathrm{Au}_{24} \\
\text { alloyed } \\
\text { nanowires }\end{array}$ & 0.49 & 0.52 & $\begin{array}{l}\text { After } 10000 \text { cycles, } \\
\text { the MA decreased to } \\
\quad 0.46 \mathrm{~A} / \mathrm{mg}_{\mathrm{Pt}}\end{array}$ & [8] \\
\hline $\mathrm{Au} / \mathrm{PtPd}$ & $\begin{array}{c}\text { Sub- } \\
\text { nanometer } \\
\text { PtPd on } \\
\text { nanoporous } \\
\mathrm{Au}\end{array}$ & 1.14 & 0.58 & $\begin{array}{c}\text { After } 100000 \\
\text { cycles, the MA } \\
\text { increased to } 1.47 \\
\mathrm{~A} / \mathrm{mg}_{\mathrm{Pt}}\end{array}$ & [9] \\
\hline $\mathrm{AuPd} / \mathrm{Pt}$ & $\begin{array}{c}\mathrm{AuPd} / \mathrm{Pt} \\
\text { core/shell } \\
\text { icosahedra }\end{array}$ & $0.98-1.85$ & $1.28-2.17$ & $\begin{array}{c}\text { After } 50000 \text { cycles, } \\
\text { the MA decreased to } \\
0.42-1.69 \mathrm{~A} / \mathrm{mg}_{\mathrm{Pt}}\end{array}$ & [10] \\
\hline
\end{tabular}

Current density @ 0.9 V vs. RHE 


\section{References}

(1) Wang, C.; van der Vliet, D.; More, K. L.; Zaluzec, N. J.; Peng, S.; Sun, S.; Daimon, H.; Wang, G.; Greeley, J.; Pearson, J.; Paulikas, A. P.; Karapetrov, G.; Strmcnik, D.; Markovic, N. M.; Stamenkovic, V. R. Multimetallic Au/ $\mathrm{FePt}_{3}$ Nanoparticles as Highly Durable Electrocatalyst. Nano Lett. 2011, 11, 919-926.

(2) Shao, M.; Peles, A.; Shoemaker, K.; Gummalla, M.; Njoki, P. N.; Luo, J.; Zhong, C. J. Enhanced Oxygen Reduction Activity of Platinum Monolayer on Gold Nanoparticles. J. Phys. Chem. Lett. 2011, 2, 67-72.

(3) Sasaki, K.; Naohara, H.; Choi, Y.; Cai, Y.; Chen, W. F.; Liu, P.; Adzic, R. R. Highly Stable Pt Monolayer on PdAu Nanoparticle Electrocatalysts for the Oxygen Reduction Reaction. Nat. Commun. 2012, 3, 1115-1123.

(4) Wang, G.; Huang, B.; Xiao, L.; Ren, Z.; Chen, H.; Wang, D.; Abruna, H. D.; Lu, J.; Zhuang, L. Pt Skin on AuCu Intermetallic Substrate: A Strategy to Maximize Pt Utilization for Fuel Cells. $J$. Am. Chem. Soc. 2014, 136, 9643-9649.

(5) Bian, T.; Zhang, H.; Jiang, Y.; Jin, C.; Wu, J.; Yang, H.; Yang, D. Epitaxial Growth of Twinned Au-Pt Core-Shell Star-Shaped Decahedra as Highly Durable Electrocatalysts. Nano Lett. 2015, 15, 7808-7815.

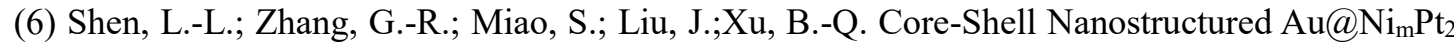
Electrocatalysts with Enhanced Activity and Durability for Oxygen Reduction Reaction. ACS Catal. 2016, 6, 1680-1690.

(7) Chen, G.; Kuttiyiel, K. A.; Su, D.; Li, M.; Wang, C.-H.; Buceta, D.; Du, C.; Gao, Y.; Yin, G.; Sasaki, K.; Vukmirovic, M. B.; Adzic, R. R. Oxygen Reduction Kinetics on Pt Monolayer Shell Highly Affected by the Structure of Bimetallic AuNi Cores. Chem. Mater. 2016, 28, 5274-5281.

(8) Chang, F.; Shan, S.; Petkov, V.; Skeete, Z.; Lu, A.; Ravid, J.; Wu, J.; Luo, J.; Yu, G.; Ren, Y.; Zhong, C. J. Composition Tunability and (111)-Dominant Facets of Ultrathin Platinum-Gold Alloy Nanowires toward Enhanced Electrocatalysis. J. Am. Chem. Soc. 2016, 138, 12166-12175.

(9) Li, J.; Yin, H.-M.; Li, X.-B.; Okunishi, E.; Shen, Y.-L.; He, J.; Tang, Z.-K.; Wang, W.-X.; Yücelen, E.; Li, C.; Gong, Y.; Gu, L.; Miao, S.; Liu, L.-M.; Luo, J.; Ding, Y. Surface Evolution of a Pt-Pd-Au Electrocatalyst for Stable Oxygen Reduction. Nat. Energy 2017, 2, 1-9.

(10) Xu, Q.; Chen, W.; Yan, Y.; Wu, Z.; Jiang, Y.; Li, J.; Bian, T.; Zhang, H.; Wu, J.; Yang, D. Multimetallic AuPd@Pd@Pt Core-Interlayer-Shell Icosahedral Electrocatalysts for Highly Efficient Oxygen Reduction Reaction. Sci. Bull. 2018, 63, 494-501. 